This item was submitted to Loughborough's Research Repository by the author.

Items in Figshare are protected by copyright, with all rights reserved, unless otherwise indicated.

\title{
Incorporating cognitive aspects in digital human modelling
}

PLEASE CITE THE PUBLISHED VERSION

https://doi.org/10.1007/978-3-642-02809-0_35

\section{PUBLISHER}

(C) Springer

\section{VERSION}

AM (Accepted Manuscript)

\section{PUBLISHER STATEMENT}

This work is made available according to the conditions of the Creative Commons Attribution-NonCommercialNoDerivatives 4.0 International (CC BY-NC-ND 4.0) licence. Full details of this licence are available at: https://creativecommons.org/licenses/by-nc-nd/4.0/

\section{LICENCE}

CC BY-NC-ND 4.0

\section{REPOSITORY RECORD}

Thorvald, Peter, Dan Hogberg, and Keith Case. 2019. "Incorporating Cognitive Aspects in Digital Human Modelling". figshare. https://hdl.handle.net/2134/26652. 
'Digital Human Modeling', Editor V.G.Duffy, pp323-332, the Proceedings of the Second International Conference on Digital Human Modeling, ICDHM 2009, Held as Part of HCI International 2009, 19-24 July 2009, San Diego, USA.

\title{
Incorporating Cognitive Aspects in Digital Human Modelling
}

\author{
Peter Thorvald ${ }^{1,2}$, Dan Högberg ${ }^{1}$ and Keith Case ${ }^{1,2}$ \\ ${ }^{1}$ University of Skövde, Skövde, Sweden \\ ${ }^{2}$ Loughborough University, Loughborough, UK \\ \{peter.thorvald, dan.hogberg\}@his.se \\ k.case@lboro.ac.uk
}

\begin{abstract}
To build software which, at the press of a button, can tell you what cognition related hazards there are within an environment or a task, is probably well into the future if it is possible at all. However, incorporating existing tools such as task analysis tools, interface design guidelines and information about general cognitive limitations in humans, could allow for greater evaluative options for cognitive ergonomics. The paper will discuss previous approaches on the subject and suggest adding design and evaluative guiding in DHM that will help a user with little to no knowledge of cognitive science, design and evaluate a human- product interaction scenario.
\end{abstract}

Keywords: Digital human modelling, cognition, context, situatedness, ecological interface design, system ergonomics.

\section{Introduction}

In Digital Human Modelling (DHM), the term ergonomics usually refers to modelling physical aspects of humans with the main focus being on anthropometry and physical strain on the body. This is also reflected in the DHM tools that exist, e.g. RAMSIS, JACK, SAMMIE, V5 Human, etc. [1, 2], tools that mainly, if not exclusively, model physical ergonomics. This paper will suggest and discuss possible ways of bringing cognition into the equation and provide users of DHM tools with an aid in evaluating cognitive as well as physical ergonomics.

Computer modelling of human cognition was originally mainly done off-line in the sense that the cognitive system is viewed as a hardware independent program, effectively disregarding the surrounding environment and even the importance of a human body. However, in later years, there has been an increasing interest for viewing the human as part of a complex system, incorporating the environment and the human body in cognitive modelling. This has led to new theories regarding how humans cognize within the world and has allowed us to regard the body and the 
context as part of the cognitive system. Human cognition is not an isolated island where we can view our surrounding context as merely a problem space. We are very much dependant on our body and our surroundings to successfully survive in the world. Previous suggestions on integrating cognition in DHM tools have largely taken its basis in symbol processing architectures such as ACT-R, Soar and such [3-5], architectures that disregard embodiment and situatedness of cognition. This paper will aim to place the computer manikins used in DHM tools within a context, a context where cognitive offloading and scaffolding onto the environment is supported.

The main advantage of using DHM and incorporating the suggested functionality is that it can be used very early in the system development process. It also allows the designer to consider the spatial information that the physical array incorporates. In traditional usability methods, this is seldom the case as design iterations are often done offline in the sense that it only incorporates some (if any) physical properties of the domain where the system is to be implemented.

\section{Cognitive Modelling in DHM}

During the last decade, there have been several attempts at incorporating cognitive modelling in DHM. A research group at Sandia National Laboratories in New Mexico have created a framework based on a modular and symbol processing view of human cognition and others have focused on a rule based system built on architectures such as ACT-R and Soar [3, 4]. Though not built on the same exact architecture, several others have gone about the problem in similar ways, ultimately trying to reach a state where the system can, at the press of a button, perform a cognitive evaluation for us [5]. However, the methodology upon which these architectures are built is challenged by researchers that recommend a more situated view on cognition as a whole. This view, originating in the 1920s from the Russian psychologist Lev Vygotsky, argues that human cognition cannot be viewed apart from its context and body [6]. There is no clear-cut line between what happens in the world and what happens in the head; the mind "leaks" into the world.

A view already seen in the DHM society is to stop dividing human factors into "neck up" and "neck down" and instead view the human as a whole [7]. This view finds much support in the work on social embodiment by Lawrence Barsalou and colleagues. They discuss how the embodiment of the self or others can elicit embodied mimicry in the self or others [8], ultimately arguing for a holistic view of the human where the body and mind are both necessary for cognition.

Whereas the discussion on embodiment and situatedness is beyond our scope in this paper, it shows us how earlier approaches to modelling cognition in DHM are at best insufficient and that a new approach is needed. The method that this paper is aimed at resulting in will not require any kind of "strong AI" and will have a much lower technological level than many others. However, it will try to consider the human as a system with a physical body, acting within an environment. 


\section{Cognition in System Ergonomics}

For a system design to become successful, the incorporation of human factors is essential. To a large part, physical ergonomics is very well accounted for in today's system design practices, but the cognizing human is often neglected. However, as technology increasingly demands more human processing abilities, the modelling of human cognition becomes more important. The range of human behaviours need to be known to design for human-related control systems [9].

System ergonomics can be used to describe a more or less complex task's mental demands on a human. It does so by three points [9].

1. Function

The main consideration of function is what the operator has in view and to what extent the task is supported by the system. It is largely defined by the temporal and spatial properties of the activities to be performed. When and where should the task be performed?

2. Feedback

The feedback allows the user to identify what state the system is in. If a performed task has resulted in anything, what task was performed etc. It is very important to allow the operator to recognize if an action had any effect on the system and also what the result of it was [10]. For example, even if a computing task on a PC takes some time to calculate, the operator is informed that the computer is working by a flashing led or an hourglass on the screen.

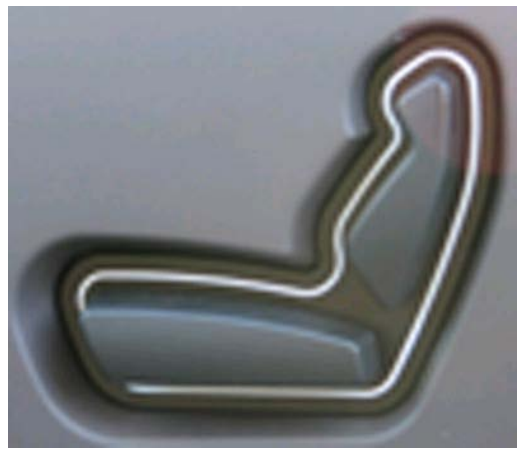

Fig. 1. A seat adjustment control which exhibits excellent natural mapping or matching between the system and the user's mental model.

3. Compatibility

Compatibility is largely about the match between systems or between the system and the user's mental model of the system. The operator should not be required to put too much effort into translating system signals. It relates information sources to each other. A very simple and obvious example from the automotive industry is described by Norman [10] with a seat adjustment control from a car. A similar seat adjustment control can be viewed in figure 1. It is obvious in the figure that the system (the adjustment control) corresponds well to the result of the task of 
manoeuvring the controls. The control maps very well to the response of the seat and to the user's probable mental model. However, the compatibility is not exclusively relevant to the psychological issues but a designer also needs to consider the physical compatibility of the user and the system. Controls might for example be spatially located away from the physical reach of the human.

Though these three points are hardly sufficient for a comprehensive design tool, they are of great help in an initial state of system design and will prove helpful to us in developing a more detailed design aid.

\section{Methods for Interface Design and Evaluation}

In human-computer interaction (HCI) there are several evaluation methods with great use for certain situations. As the aim of this paper is to present a draft of a design tool, we shall take a closer look at a few of these methods along with a task analysis tool.

\subsection{Task Analysis}

All good design processes include some sort of task analysis. To be able to design a system that fits both task and human, we need to know as much as possible about the task. A fairly quick and dirty task analysis which provides a good basis for further development is the hierarchical task analysis (HTA) [11]. A HTA is a tree diagram of the task structure and serves several purposes. It gives us a good overview of the system or the task and subtasks that need to be performed, and provides aid in achieving common ground within a design group. It can also even serve as a task evaluation tool, allowing a designer to find global problems that can be missed while using usability inspection methods such as cognitive walkthrough [12], heuristic evaluation $[13,14]$ etc. Global issues are mainly related to the structure of the task and the relation between the subtasks whereas local issues are within a subtask with a very limited scope.

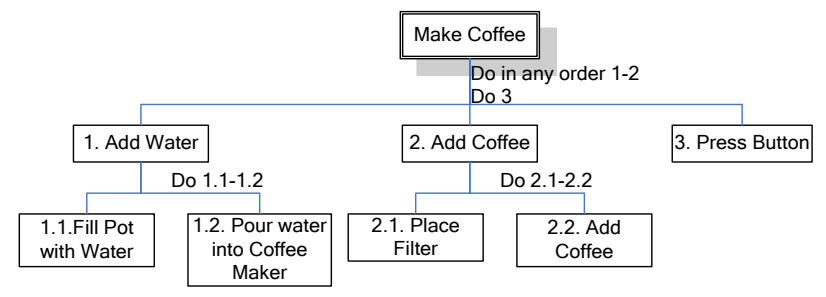

Fig. 2. A very simple HTA of the process of making a pot of coffee.

The creation of a HTA is fairly simple. First, identify the overlying task to be performed, which in our very simple example, illustrated in figure 2, is making a pot of coffee. The HTA in figure 2 shows this process and also shown are the plans within 
which each subtask should be performed. In this example it is limited to doing the tasks in order or doing two subtasks first in any order and then continuing with the third. However, these plans can be pretty much anything you want them to be such as selections (do one but not the other), linear or non- linear, or even based on a specific condition (if $\mathrm{X}$ then do $\mathrm{Y}$, else $\mathrm{Z}$ ).

The finished task analysis is then used as a basis for further inspections and design iterations.

\subsection{Ecological Interface Design}

Ecological interface design (EID) is spawned from cognitive work analysis (CWA), which was developed as an analytical approach to cognitive engineering by the Risø group in Denmark [15]. CWA was developed to aid in the design of very critical human-machine systems such as nuclear power plant control rooms to make them safer and more reliable. It is an approach that allows the operator to handle situations that the system designers had not anticipated. CWA is made up of five phases to analyze within a system. These phases are work domain analysis, control task analysis, strategies analysis, social-organisational analysis and worker competencies analysis [16]. Having these analyses allows the designer and the operator a better understanding of the system and already this enables the operator to better respond to unforeseen events.

The idea behind EID is to create interfaces based on certain principles of CWA. It is very closely related to the principles of ecological psychology and direct perception, concepts developed by J.J Gibson in the 70's [17]. Gibson argued that there is enough information in the visual array to directly perceive information and that mental processing of visual information is not necessary. Though this claim is highly challenged, EID is largely built up around these principles in that its goal is to create interfaces containing objects that visually reveal information on their function. A related goal of EID is to make affordances visible in interface design. Affordances, another concept created by Gibson, are the action possibilities of a specific object [17, 18].

The ideas surrounding affordances and EID can also be found in other areas of the scientific literature. In product design, one tends to discuss similar issues in terms of semantics [19].

\subsection{Usability Inspections}

Usability inspection methods are predictive evaluation methods, usually performed without end user participation (although this is not a prerequisite). Usability experts simulate the users and inspect the interface resulting in problem lists with varying degree of severity [20].

\subsubsection{Cognitive Walkthrough}

A cognitive walkthrough is usually performed by usability experts considering, in sequence, all actions incorporated in a predefined task. Its focus is almost exclusively 
on ease of learning, not taking into account first time problems, problems that might not be a problem for the experienced user. The method contains two phases. First the preparations phase where the analyst defines the users, their experience and knowledge; defines the task to be analyzed; identifies the correct sequence of actions to achieve the goal of the task. In the second phase, the analysis phase, the analyst answers and motivates a set of questions for each action within the task [12].

1. Will the user try to achieve the right effect? For example, if the task is to fill up the car with petrol and a button first has to be pressed from inside the car to open the gas cap, does the user know that this has to be done?

2. Will the user notice that the correct action is available? Simply pressing the button for the gas cap would not be a problem but if the button has to be slid or twisted in some way the user may not think of this.

3. Will the user associate the correct action with the desired effect? Is it clear that this is what the specific control is for? Unambiguous icons and names of controls are important to this aspect.

4. If the correct action is performed, will the user see that progress is being made? The importance of feedback, discussed earlier, comes into play here.

These questions, though applicable to many tasks, are merely guidelines towards conducting a successful cognitive walkthrough. The method's advantages are its focus on detail, it identifies local problems within the task and considers the users' previous knowledge and experiences. However, it rarely catches global problems related to the overlying structure of the task and can be viewed as fairly subjective. It also requires a detailed prototype for evaluation although this would probably not be a problem if it is complementing a DHM-tool where a virtual prototype is likely to already exist.

\subsubsection{Heuristic Evaluation}

Just as in the case of cognitive walkthrough, heuristic evaluations are usually performed by usability experts sequentially going through each action within a main task with a basis in a set of heuristics [14]. The method is developed by usability expert Jacob Nielsen and a set of his heuristics can be found through his publications [13, 14, 21]. Examples of Nielsen's heuristics are

\section{- Match between system and the real world}

o Similar to the matching and mapping concept discussed in system ergonomics, the system should speak the users' language, matching the real world in terms of terminology and semiotics.

- Consistency and standards

o Also related to the matching concept is using accepted conventions to avoid making users wonder whether different words, icons or actions mean the same thing in different contexts.

- Recognition rather than recall

o Options should be made visible to avoid making the user have to remember how or where specific actions should be performed.

- Aesthetic and minimalist design 
o Dialogues and controls should not be littered with irrelevant or rarely used information.

Heuristics can be added and subtracted to fit certain tasks before the evaluation commences. The method results in problem lists with motivations and rankings of the severity of the problems found.

\section{A Design Guide for DHM}

The evaluation and design tools discussed in previous sections are developed for interface design in different settings than DHM. However, the design guide suggested in this section is a hybrid of these, adapted for use under the specific conditions that DHM provides. The method will strive to take into account global as well as local issues through the use of action based interface inspections and a task analysis focusing on the structure of the task.

As stated earlier in this paper and by others [22], every good design process starts with a task analysis. For our purposes, a hierarchical task analysis is very suitable as it complements the inspection methods incorporated in this design guide. The HTA will serve several purposes; it will give the designer a better understanding of the task and it will provide a common understanding of the task within a development group. The task analysis can also be used as an evaluation tool of the task itself. It allows the designer to identify problems in the task structure that could result in problems with automatism [23], it can identify recurring tasks and give them a higher priority in the interface etc. Complementary to the task analysis, the designer should consider who the users are and what a priori knowledge they have. This resembles the guiding system for utilising traditional DHM tools in development processes suggested by Hanson et al. [24], where the users' anthropometry and tasks are defined before the actual analyses or simulations are performed.

The sequence-based walkthrough will take its basis in the task analysis performed. For each subtask (box) of the HTA, a set of questions, based on Bubb's points regarding system ergonomics [9], will act as guidelines for the design.

- Function - When and where should the action be performed?

o Will the user identify the action space where the correct action should be performed? What do the physical and geographical properties of each control convey to the user?

o Frequency of actions - a frequently recurring action should take precedence in taking up place and intrusiveness in the physical and cognitive envelope.

o Importance of action - Safety critical systems should also take precedence in the available information space.

o Minimalism of design - avoid taking up space with irrelevant or rarely needed information. Hick's law: Reaction time is a function of the number of choices in a decision [25].

- Feedback 
o Will the user understand that a correct or faulty move has been made?

o Is the system status visible?

\section{- Compatibility}

o Does the system match other, similar systems in terms of semantics, semiotics etc.?

o Does the system match the real world and the plausible mental model of the user?

o Are demands on consistency and standards of the domain met?

o Action-effect discrepancies - is it obvious beforehand that a certain action will have a certain effect?

\subsection{Function}

In figure 3, there is an example of what a virtual interface, modelled in a DHM-tool can look like. In this case the picture shows a fighter jet cockpit used for evaluation where the pilot needed to locate a "panic button" to bring the aircraft back into control under extreme physical and mental conditions.

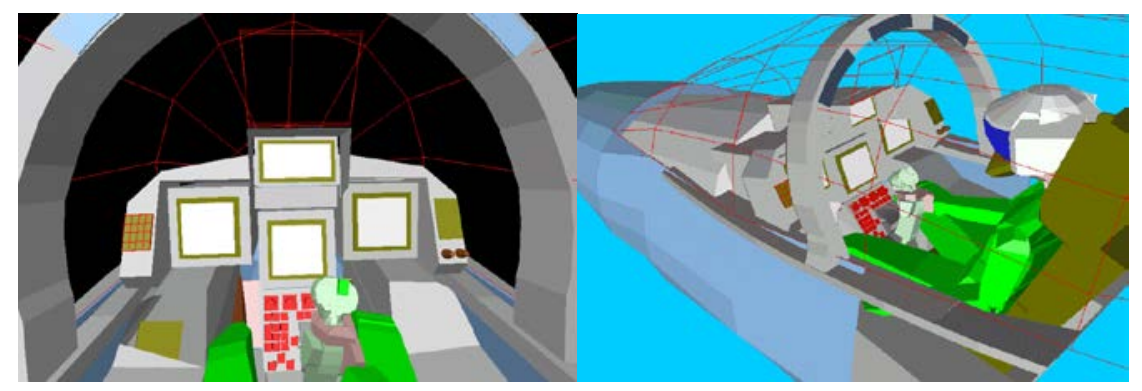

Fig. 3. Two views of a cockpit modelled in SAMMIE.

The action spaces that the user has to identify when performing an action are the controls in front of, and to the right and left of the steering control stick. Preferably, a frequently performed action control should be placed on the control stick or directly in front of it as these are the spaces that best correspond to the physical and cognitive reach of the pilot. Also safety systems, as in the case of the evaluation in figure 3, should be placed so that they are easily accessible for the user. Knowing that certain controls are rarely used, they can be placed to the right and left to avoid having too many options in terms of pushable buttons at the same place. The intrusiveness and affordances of such "high priority controls" should also be accentuated in terms of their design.

\subsection{Feedback}

Understanding what has been done and what is in progress of happening with the system can prove vital in many cases. Surely we can all relate to a situation where we have pressed the print button more than once only to find out that we have printed 
several more copies than needed. While this may be a minor problem, one can easily imagine the problems that can arise in more critical domains. What if there were no indications for what gear the car's gearbox was in? The driver would have to test each time to see if the car is in reverse or drive. In an incident at a hospital, a patient died as a result of being exposed to a massive overdose of radiation during a radiotherapy session. The problem could easily have been avoided, had the system provided the treating radiology technician with information of the machines settings [26].

\subsection{Compatibility}

Accurate mapping between systems and mental models is a key concept in the compatibility section. This includes trying to adhere to consistencies and standards of the organisation and the specific field. There should also be clear connection between action and effect. Neglecting these consistencies can lead to serious problems as in the case with an aircraft's rudder settings. The sensitivity of the rudder could be set through a lever placed to the side of the pilot's seat. However, between the simulator for the aircraft and the actual aircraft, the lever was reversed, moving in the opposite direction for maximum and minimum sensitivity almost resulting in a crash [27].

\section{Conclusions \& Future Work}

In ergonomics, it seems to be common practice to separate human factors into "neck up" and "neck down". Though this approach may make it easier to study ergonomics, it does not portray an entirely accurate picture of the human. The evidence for a tight coupling between mind and body is so overwhelming that instead of talking about mind and body, perhaps we should be talking about the human system.

The aim of this paper has been to consider past and current approaches towards integrating cognition into DHM tools and outline a new design guide to help designer achieve this integration in a better way. The guide is not complete and would need extensive further development and testing. However, it is a good start towards including something more into DHM than traditionally has been found there.

\section{References}

1. Bubb, H.: Future Applications of DHM in Ergonomic Design. LECTURE NOTES IN COMPUTER SCIENCE 4561 (2007) 779

2. Case, K., Porter, J.M.: SAMMIE - A Computer Aided Ergonomics Design System. Engineering 220 (1980) 21-25

3. Bernard, M.L., Xavier, P., Wolfenbarger, P., Hart, D., Waymire, R., Glickman, M., Gardner, M.: Psychologically Plausible Cognitive Models for Simulating Interactive Human Behaviors. Proceedings of the Human Factors and Ergonomics Society 49th Annual Meeting (2005) 1205-1210 
4. Carruth, D.W., Thomas, M.D., Robbins, B., Morais, A.: Integrating Perception, Cognition and Action for Digital Human Modelling. In: Duffy, V.G. (ed.): Digital Human Modelling, HCII 2007, Vol. LNCS 4561. Springer-Verlag, Berlin (2007) 333-342

5. Gore, B.F.: Human Performance: Evaluating the Cognitive Aspects. In: Duffy, V.G. (ed.): Handbook of digital human modelling, Mahwah, New Jersey (2006)

6. Clark, A.: Being There: Putting Brain, Body, and World Together Again. MIT Press (1997)

7. Feyen, R.: Bridging the Gap: Exploring Interactions Between Digital Human Models and Cognitive Models. In: Duffy, V.G. (ed.): Digital Human Modelling, HCII 2007, Vol. LNCS 4561. Springer-Verlag, Berlin (2007) 382-391

8. Barsalou, L.W., Niedenthal, P.M., Barbey, A.K., Ruppert, J.A.: Social Embodiment. In: Ross, B.H. (ed.): The Psychology of Learning and Motivation, Vol. 43-92. Academic Press, San Diego, CA (2003)

9. Bubb, H.: Computer Aided Tools of Ergonomics and System Design. Human Factors and Ergonomics in Manufacturing 12 (2002) 249-265

10.Norman, D.A.: The design of everyday things. Basic Books New York (2002)

11.Annett, J.: Hierarchichal Task Analysis. In: Diaper, D., Stanton, N. (eds.): The Handbook of Task Analysis for Human-Computer Interaction. Lawrence Erlbaum Associates, Mahwah, New Jersey (2003) 67-82

12.Polson, P.G., Lewis, C., Rieman, J., Wharton, C.: Cognitive Walkthroughs: A Method for Theory-Based Evaluation of User Interfaces. International Journal of Man-Machine Studies 36 (1992) 741-773

13.Nielsen, J.: Usability Engineering. Morgan Kaufmann (1993)

14.Nielsen, J.: Heuristic evaluation. In: Nielsen, J., Mack, R.L. (eds.): Usability inspection methods. John Wiley \& Sons, Inc., New York (1994) 25-62

15.Vicente, K.J.: Cognitive Work Analysis: Toward Safe, Productive, and Healthy ComputerBased Work. Lawrence Erlbaum Assoc Inc (1999)

16.Sanderson, P.M.: Cognitive work analysis. In: Carroll, J.M. (ed.): HCI models, theories, and frameworks: Toward an interdisciplinary science. New York: Morgan-Kaufmann. Morgan Kaufmann Publishers, San Francisco, USA (2003) 225-264

17.Gibson, J.J.: The ecological approach to visual perception. Lawrence Erlbaum Associates., Hillsdale, NJ (1986)

18.McGrenere, J., Ho, W.: Affordances: Clarifying and Evolving a Concept. Proceedings of Graphics Interface 2000 (2000) 179-186

19.Monö, R.: Design for Product Understanding. Skogs Boktryckeri AB (1997)

20.Nielsen, J., Mack, R.L.: Usability inspection methods. Wiley New York (1994)

21.Nielsen, J.: Finding usability problems through heuristic evaluation. Proceedings of ACM, Monterey, CA (1992) 373-380

22.Pheasant, S., Haslegrave, C.M.: Bodyspace: Anthropometry, Ergonomics and the Design of Work. CRC Press (2006)

23.Thorvald, P., Bäckstrand, G., Högberg, D., de Vin, L.J., Case, K.: Demands on Technology from a Human Automatism Perspective in Manual Assembly. Proceedings of FAIM2008 Vol. 1, Skövde, Sweden (2008) 632-638

24.Hanson, L., Blomé, M., Dukic, T., Högberg, D.: Guide and documentation system to support digital human modelling applications. International Journal of Industrial Ergonomics 36 (2006) 17-24

25.Hick, W.E.: On the rate of gain of information. The Quarterly Journal of Experimental Psychology 4 (1952) 11-26

26.Casey, S.M.: Set phasers on stun and other true tales of design, technology, and human error. Aegean, Santa Barbara, CA (1998)

27.Casey, S.M.: The atomic chef : and other true tales of design, technology, and human error. Aegean Pub. Co., Santa Barbara, CA (2006) 